\title{
Numerical study of the electron lateral distribution in atmospheric showers of high energy cosmic rays
}

\author{
Georgios Atreidis, Anastasios Liolios \\ Physics Department, Aristotle University of Thessaloniki, 54124 Thessaloniki, Greece
}

Email address:

gatridis@physics.auth.gr (G. Atreidis), lioliosa@auth.gr (A. Liolios)

\section{To cite this article:}

Georgios Atreidis, Anastasios Liolios. Numerical Study of the Electron Lateral Distribution in Atmospheric Showers of High Energy Cosmic Rays. American Journal of Modern Physics. Vol. 2, No. 6, 2013, pp. 287-291. doi: 10.11648/j.ajmp.20130206.13

\begin{abstract}
The study of the lateral distribution of electrons in an atmospheric cascade can lead to useful conclusions on the mass and energy of the primary cosmic particle. In this work we compare the results we get from simulations by the CORSIKA program, with the theoretical NKG function expressing the lateral distribution of electrons. We then modify the original NKG function to fit better the data of the simulation. Finally, we propose this procedure as a method to approach the mass of the primary particle which started the atmospheric cascade.
\end{abstract}

Keywords: Cosmic Rays, Lateral Distribution, Atmospheric Cascade, Atmospheric Shower

\section{Introduction}

The nature of cosmic rays at very high energies is surrounded by uncertainties. The variation of their composition with energy plays an important role in the understanding of galactic and extragalactic cosmic rays.

The determination of the mass of the cosmic particles, with energies greater than $10^{7} \mathrm{GeV}$, can give important clues for the origin of cosmic rays of very high energy.

However, at very high energies the mass of cosmic rays cannot be measured directly. In order to study the cosmic particles at very high energy, it is necessary to study the characteristics of the distribution of secondary particles created from a primary particle in the atmosphere. The secondary particles are distributed over a large area around the propagation direction of the original particle. The disc of the secondary particles can extend over several hundred meters from the axis of the cascade with the highest density in the center of the disc.

A study of the secondary particles, such as the lateral distribution of their density in an extended cascade, brings important information for the development of the cascade and the nature of the primary particle. The NKG (Nishimura-Kamata-Greisen) function gives a good theoretical approach for the lateral distribution of electrons [1]. The lateral growth of the cascade depends on the characteristics of the high energy interactions and the type of the primary particle. The effect of the primary particle to the growth of the cascade into the atmosphere is studied in the present work with the analysis of the lateral distribution of electrons for cascades having for primary particle: proton, photon, and nuclei of helium, oxygen and iron.

\section{The Simulation}

\subsection{Input Data}

The Monte Carlo technique is used for the simulation of the lateral growth of an extensive air shower which has a specified primary particle type and energy corresponding to a specific interaction model. The CORSIKA (COsmic Ray SImulations for KAscade) is a Monte Carlo simulation program for the physics of cosmic rays, used by many cosmic ray experiments for various actions. It can be used to simulate interactions and decays of nuclei, hadrons, muons, electrons, and photons in the atmosphere up to energies of some $10^{20} \mathrm{eV}$. It gives details for all secondary particles that are created in an air shower and pass a selected observation level [2].

In this paper, for each entry angle we ran 100 simulations with fixed primary particle energy and of zenith angles varying from 0 to 75 degrees. The results obtained as the mean value of all simulations for each particle. The corresponding errors are due to the limited number of events in the Monte Carlo simulation.

In our simulation we used the 6990 version of CORSIKA and we chose QGSJET (Quark Gluon String model with JETs) that is a program developed to describe high energy 
hadronic interactions [3]. The cutoff energy for the particles, i.e. the energy under which the program stops giving the particle interactions, was $0.3 \mathrm{GeV}$ (hadrons, muons) and $0.015 \mathrm{GeV}$ (electrons, photons).

The lateral distribution of the electrons has been measured from the core of the cascade at an altitude of 110 $\mathrm{m}$ above sea level (observation level).

The first section of the present study was made for particles with primary energy $10^{7} \mathrm{GeV}$ (proton, photon and iron nuclei) and for different zenith angles $\theta$. The azimuth angle was kept constant $(\varphi=0$ degrees, $\varphi=20$ degrees and $\varphi=60$ degrees).

The second section of the present study was made for particles with primary energy $10^{7} \mathrm{GeV}$ (proton and nuclei of helium, oxygen, silicon, calcium and iron) and for entry zenith angle 20 degrees. The azimuth angle was kept constant ( $\varphi=0$ degrees) in all cascades.

In this section, we modified the NKG function to fit best to our results and drew conclusions about the dependence of the exponent $\mathrm{s}$ from the mass of the primary particle.

\subsection{Results}

Compare of the lateral distribution of electrons for different primary particle and at different angles.

When the entry angle $\theta$ is small $0^{\circ}<\theta<20^{\circ}$ the differences of lateral distribution (of electrons arriving at the observation plane and the electrons arriving at the plane perpendicular to the axis of the cascade) are small. But when the entry angle increases, some electrons arrive at the observation level (Earth's surface) by the shortest path (point $\mathrm{C}$ in Figure 1), while others arrive from the longest path (point $\mathrm{A}$ in Figure 1). The cascade development on the short path is faster. In the long path we have greater absorption of electrons, thus the larger the entry angle; the fewer electrons reach at point $\mathrm{A}$.

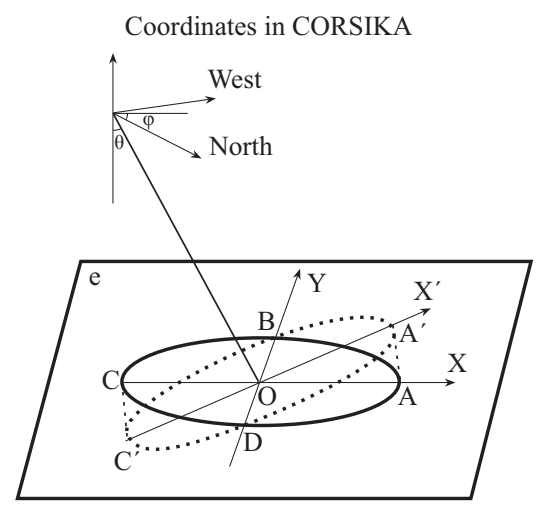

Figure 1. The plane e is the level of observation. The lateral distribution of electrons is symmetrical to the dashed plane which is perpendicular to the axis of the cascade [4].

The lateral distribution of electrons for primary particle proton, photon and iron nuclei, for 0 degrees entry angle is shown in Figure 2. Respective Figures derived (according to the simulation) for small entry angles $0^{\circ}<\theta<20^{\circ}$.

But in all the scenarios of the simulation where the primary particle is photon (electromagnetic cascade), the density of electrons is greater.

The diagram of Figure 2 shows that there is symmetry around the center of the cascade (in a plane perpendicular to the axis of the cascade).

When the entry angle is small $\theta<20$ degrees, the plane which is perpendicular to the axis of the cascade and the observation plane are almost identical. But as the entry angle increases, the angle of the two planes (Figure 1) increases respectively. Thus there is no longer symmetry on both sides of the axis of the cascade at the level of observation.

The variation in the number of lateral electron explained by the difference of the two roads $\mathrm{d}=\mathrm{AA}^{\prime}+\mathrm{CC}^{\prime}=2 \mathrm{CC}^{\prime}=$ $2 x \tan \theta$ (for angle $\theta=60$ degrees), followed by the electrons in the cascade. Thus, the lateral distribution of electrons is not symmetrical (Figure 3) [5].

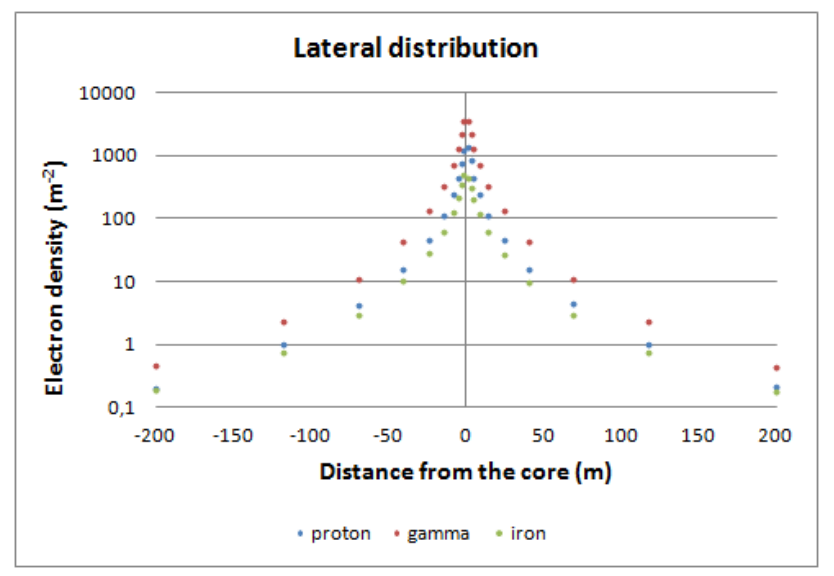

Figure 2. Lateral distribution of electrons for cascades of primary particles proton, photon and iron nuclei with primary energy $10^{7} \mathrm{GeV}$ and entry angle 0 degrees.

What causes this?

When increasing the entry angle, the cascade develops into greater heights, since we have more lateral route to the higher layers of the atmosphere. So the electrons arriving at observation level are significantly less.

But over 40 degrees and apparently over 60 degrees the data differ in the symmetry of the lateral distribution. The electrons that reach the observation level from the long path are less than those arriving from the small path (Figure $3)$.

In very large zenith angles $\theta \sim 90$ degrees, cosmic rays develop their own cascades in the upper atmosphere, with a similar mechanism as that in the vertical cascades. Their electromagnetic component almost completely absorbed by the increased atmospheric side depth and cannot reach the ground. A few electrons with irregular distribution reach the Earth's surface from only one side of the cascade [6].

In contrast, the high energy neutrinos can cause the creation of horizontal cascades at a larger depth in the atmosphere and therefore likened to vertical atmospheric cascades as to the particles that reach the ground. 


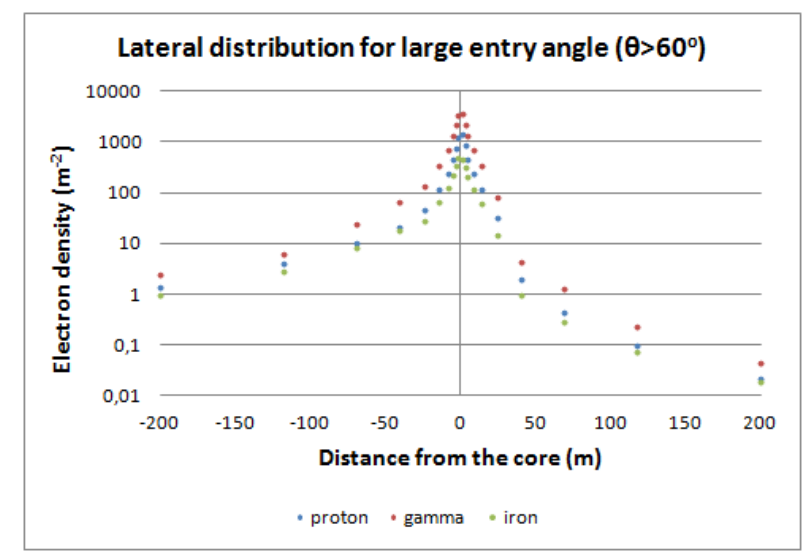

Figure 3. In large entry angles $\theta>60^{\circ}$ for electrons arriving at observation level there is no symmetry. The cascade develops differently in the lateral plane.

So the assumption that we can detect high energy neutrinos by horizontal cascades takes on a consistent basis.

Compare of the lateral distribution of electrons on the same original particle and at different angles.

We applied the simulation for a proton primary particle with energy $10^{7} \mathrm{GeV}$ and for different entry angles. By increasing the entry angle, the number of electrons that reach the ground decreased, accordingly to the reasons mentioned above.

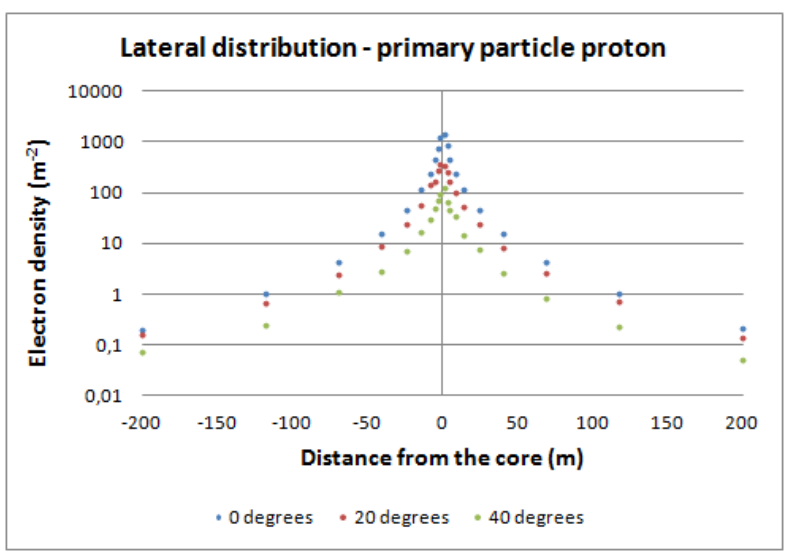

Figure 4. Lateral distribution of electrons for cascades with a proton primary particle with primary energy $10^{7} \mathrm{GeV}$ and entry angles 0,20 and 40 degrees.

Another reason for the reduction of the lateral electron is the height of the first interaction. Obviously in large entry angles that height increases since the initial particle travels deep side to first interaction.

\section{Configuration of the Lateral Distribution of Electrons}

\subsection{The NKG function}

The atmospheric cascade is a superposition of individual cascades generating electrons (those that concern our work), and muons at different depths and with different energies. The NKG (Nishimura-Kamata-Greisen) function is a good theoretical approach to the lateral distribution of electrons $[1,7]$.

This function, which is adapted to the electrons, is given by the formula below.

$$
\rho(r)=N_{e} C(s)\left(\frac{r}{r_{M}}\right)^{s-2}\left(1+\frac{r}{r_{M}}\right)^{s-4.5}
$$

Where $\mathrm{C}(\mathrm{s})$ is normalization constant, $\mathrm{r}_{\mathrm{M}}$ is the Moliere radius (for electrons the Moliere radius is $80 \mathrm{~m}$ in the atmosphere) and $\mathrm{s}$ is the time slope parameter (age parameter). The constant $\mathrm{C}(\mathrm{s})$ is given by the relation below.

$$
\mathrm{C}(\mathrm{s})=\frac{\Gamma(4,5-\mathrm{s})}{2 \pi \mathrm{r}_{\mathrm{M}}^{2} \Gamma(\mathrm{s}) \Gamma(4,5-2 \mathrm{~s})}
$$

The above function is analytically derived for the case of electromagnetic cascade but is also used in the description of the lateral distribution of electrons in a hadronic cascade. However, based on knowledge of previews studies, it seems that the NKG functions have shortcomings in the implementation of the measured lateral distribution of electrons, becoming more evident in the large distances from the axis of the cascade.

This deficit is usually caused by the fact that the NKG function has occurred for electromagnetic cascades while hadronic cascades are a superposition of a large number of independent electromagnetic cascades. For typical cascade, detectors extend in approximately $200 \mathrm{~m}$ from the axis of the cascade.

In the case of large cascades with over $10^{6}$ electrons with primary energy $10^{16} \mathrm{eV}$, detectors near the axis of the cascade are saturated and must be removed from the analysis.

So the lateral distribution differs significantly for small and large cascades in both (upper and lower) bounds. The deviation of the NKG function from the actual shape of the lateral distribution of electrons gives systematic errors to the lateral shape parameter (age parameter s) which depends on the scale of the lateral adjustment that we do, based on our data and therefore on the size of the cascade.

It has been noted that the determination of the time slope parameter $s$, instead of the change of parameter $r_{M}$ significantly improves the behavior of the NKG function adjustment [8].

The above definition works well only for average values of the lateral distribution obtained from a large number of cascades. During the adjustment of individual events which have large statistics and physical variations between the stations of detectors, this method proved significantly unstable. Especially for small cascades it leads to unreliable results $[9,10]$.

Another way that improves the imperfections of the NKG function describing the lateral distribution of 
electrons in a hadronic cascade is to replace it with a different function, or change the form by changing the values of the exponents. So we can get a better adjustment to the shape of the lateral distribution.

\subsection{Reconstruction of Cosmic Rays for the Determination of their Mass}

The results obtained from the simulation of atmospheric cascades with CORSIKA lead us to the modification of the NKG function by varying the exponent values. The new function which fits better the results of the simulation than the original NKG function is:

$$
\rho=C\left(\frac{r}{r_{o}}\right)^{s-1,8}\left(1+\frac{r}{r_{o}}\right)^{2 s-3,8}
$$

where the constant $\mathrm{C}$ is

$$
\mathrm{C}=\frac{\mathrm{N}_{\mathrm{e}}}{2 \pi \mathrm{r}_{\mathrm{o}}^{2}} \frac{\Gamma(3,8-\mathrm{s})}{\Gamma(\mathrm{s}+0,2) \Gamma(3,6-2 \mathrm{~s})}
$$

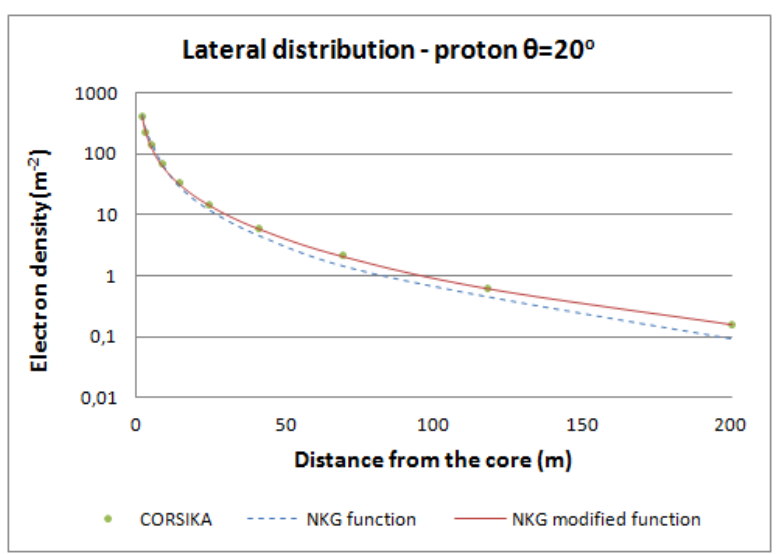

Figure 5. Lateral distribution of electrons for cascades with a primary particle proton having primary energy $10^{7} \mathrm{GeV}$ and entry angle 20 degrees. The solid line represents the fitted function while the dashed represents the original.

We study cascades with primary particle proton, helium, oxygen, silicon, calcium and iron. The primary particle energy is $10^{16} \mathrm{eV}$. The entry angle for each particle is the 20 degrees. Figure 5 shows the fitting of the original and the modified NKG function.

The table 1 gives the exponent $\mathrm{s}$ obtained as the mean value of all simulations for each particle. The corresponding errors are due to the limited number of events in the Monte Carlo simulation.

Table 1. Values of the exponent s

\begin{tabular}{cc}
\hline Particle - Nucleus & exponent s \\
\hline proton & $0,75 \pm 0,06$ \\
helium & $0,63 \pm 0,06$ \\
oxygen & $0,79 \pm 0,06$ \\
silicon & $0,85 \pm 0,06$ \\
calcium & $0,89 \pm 0,06$ \\
iron & $0,90 \pm 0,06$ \\
\hline
\end{tabular}

Based on the values of the exponent $s$ we can draw conclusions about the mass of the primary particle.

The variation of the exponent s depending on the mass is shown on the diagram in Figure 6.

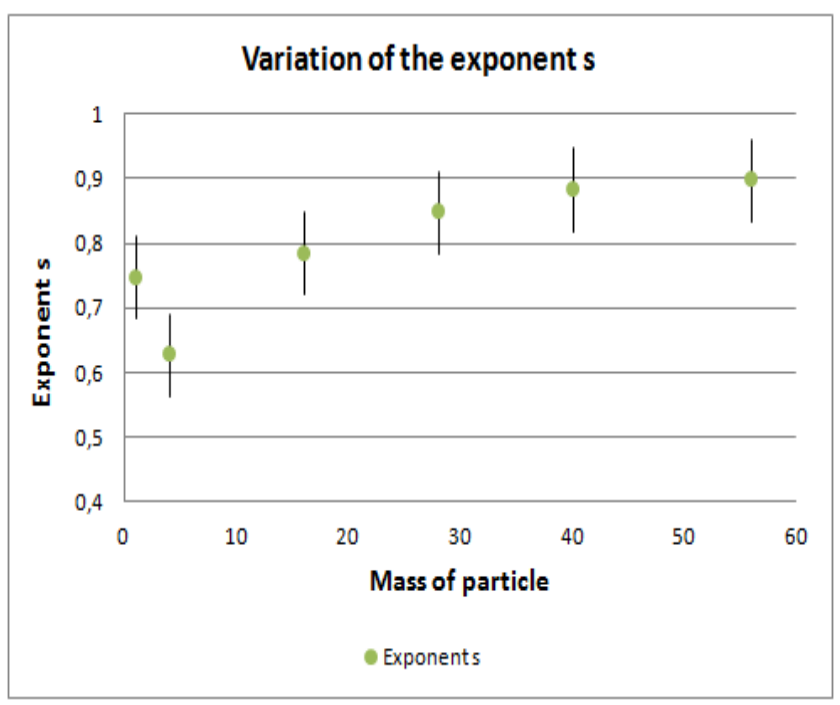

Figure 6. Variation of the exponent s, in relation to the mass of the primary particle.

\section{Conclusions}

Studying the data of our simulation we can reach the following conclusions.

There is symmetry in the density of the lateral distribution of the electrons in relation to the axis of the cascade, as shown in Figure 2. For heavier primary nuclei the electron density decreases but the symmetry remains. But as the entry angle increases, the symmetry on a plane perpendicular to the axis of the cascade remains, while on the observation plane there is no longer symmetry on both sides of the axis of the cascade (Fig. 3 ).

The lateral electron density decreases for larger entry angles as shown in Figure 4. This is due to the higher absorption of electrons because of the lateral distance traveled until they reach the ground as well as the greatest height of the first interaction.

The initial NKG function describes well the lateral distribution of electrons in smaller distances from the core of the cascade. For larger distances we propose a modified NKG function. With this modified function we can achieve better fit also at the greater distances from the core of the cascade.

Finding the parameter $\mathrm{s}$ of the modified NKG function that adjusts the simulation data better, we can make conclusions for the primary mass of the particle. It appears that the exponent $\mathrm{s}$ varies with the mass of the primary particle up to $Z \approx 18$, remaining nearly constant for heavier nuclei.

Our aim is to extend this study for larger primary energies and larger entry angles, where the lateral distribution of electrons seems to be completely asymmetrical in relation to the core of the cascade 


\section{References}

[1] W.D. Apel et al., "Comparison of measured and simulated lateral distributions for electrons and muons with KASCADE," Astroparticle Physics 24 (2006) 467-483

[2] D. Heck and T. Pierog, "Extensive Air Shower Simulation with CORSIKA, A User's Guide", Karlsruher Institut für Technologie, 2013

[3] S. Ostapchenko, "QGSJET-II: towards reliable description of very high energy hadronic interactions", Nuclear Physics B (Proc. Suppl.) 151 (2006) 143-146

[4] O. Sima et al., "Restoring the azimuthal symmetry of lateral distributions of charged particles in the range of the KASCADE - Grande experiment", Nuclear Instruments and Methods in Physics Research A638 (2011) 147-156

[5] D. Kalita, K.Boruah, "Study of Lateral distribution Parameters from simulation of HE Cosmic Ray EAS," $32^{\text {nd }}$ Int. Cosmic Ray Conf., Vol. 1 (2011) 247-250

[6] M. Oliveira, "Measurement of the Number of Muons in Inclined Showers at the Pierre Auger Observatory," Thesis, Lisbon Univ., Portugal, 2012

[7] J. Chang et al., "An excess of cosmic ray electrons at energies of 300-800 GeV," Nature 456 (2008) 362-365

[8] A. Geranios, et al., "Energy Estimation of Ultra High Energy Cosmic Hadrons and Gamma Rays by Lateral Distribution Functions of Extensive Air Showers" $31^{\text {st }}$ Int. Cosmic Ray Conf. (2009) 1

[9] T. Antoni et al. (KASCADE Collaboration), "Electron, muon and hadron lateral distributions measured in air showers by the KASCADE experiment," Astroparticle Physics 14, (2001) 245-260

[10] P. Sommers University of Utah, "Experimental aspects of cosmic rays," Univ. of Utah, Salt Lake City, (2008), http://cds.cern.ch/record/1017823/files/p177.pdf 